\title{
COMPORTAMENTO DOS CUSTOS: UMA INVESTIGAÇÃO EMPÍRICA ACERCA DOS CONCEITOS ECONOMÉTRICOS SOBRE A TEORIA TRADICIONAL DA CONTABILIDADE DE CUSTOS*
}

\author{
COST BEHAVIOR: AN EMPIRICAL STUDY OF ECONOMETRIC \\ CONCEPTS ABOUT TRADITIONAL COST ACCOUNTING THEORY
}

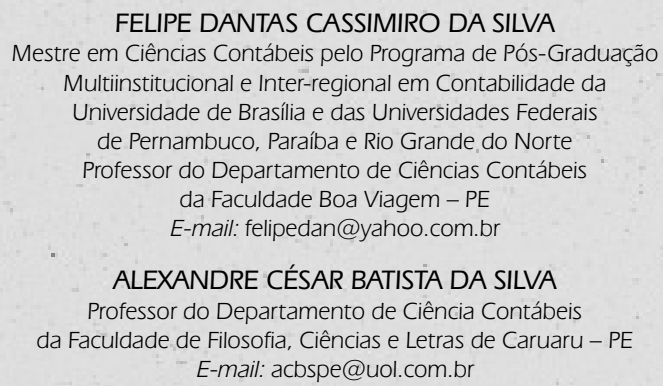

FELIPE DANTAS CASSIMIRO DA SILVA

Mestre em Ciências Contábeis pelo Programa de Pós-Graduação

Multiinstitucional e Inter-regional em Contabilidade da

Universidade de Brasilia e das Universidades Federais

de Pernambuco, Paraíba e Rio Grande do Norte

Professor do Departamento de Ciências Contábeis

da Faculdade Boa Viagem - PE

E-mail: felipedan@yahoo.com.br

ALEXANDRE CÉSAR BATISTA DA SILVA

Professor do Departamento de Ciência Contábeis

da Faculdade de Filosofia, Ciências e Letras de Caruaru - PE E-mail: acbspe@uol.com.br

\author{
MARCO TÚLIO DE CASTRO VASCONCELOS \\ Professor Adjunto 3 do Departamento de Ciências Contábeis \\ do Centro de Ciências Sociais Aplicadas \\ da Universidade Federal de Pernambuco - PE \\ E-mail:mtullio@proplan.ufpe.br \\ SEBASTIÃO MARCOS CAMPELO \\ Mestrando do Programa de Pós-Graduação Multiinstitucional \\ e Inter-regional em Contabilidade da Universidade de Brasília \\ e das Universidades Federais de Pernambuco, Paraíba e \\ Rio Grande do Norte \\ E-mail: campelo@trf5.gov.br
}

\section{RESUMO}

A investigação, em tela, versa sobre a inconsistência dada pela literatura Contábil em termos de estimação do comportamento dos custos. Portanto, o objetivo central deste trabalho é identificar se teoria da Análise Estatística do Comportamento dos Custos, defendida pela literatura contábil, está ferindo os conceitos modernos da Econometria utilizados em finanças, em termos de identificação e correção da não estacionariedade das séries temporais. Para tanto, adotou-se um procedimento metodológico dedutivo através de pesquisa indireta. Para reforçar os conceitos e análises feitas no desenvolvimento da pesquisa utilizaram-se dados de estudo de caso que foram rodados no software Econométrico E-views. Por fim, conclui-se que a teoria empregada na literatura contábil concernente à utilização de técnicas estatísticas em comportamento dos custos não considera a análise da estacionariedade das séries e que, portanto, gera inconsistências estatísticas (regressões espúrias) na previsão do referido comportamento. Com isso, o estudo posiciona-se de forma relevante à evolução da ciência no momento em que enseja uma discussão maior em uma área ainda incipiente das Ciências Contábeis, a Contabilometria.

Palavras-chave: Contabilidade de Custos; Contabilometria; Estacionariedade.

\section{ABSTRACT}

This research looks at the inconsistency in accounting literature about the estimation of cost behavior. The main goal was to identify whether the Statistical Analysis of Cost Behavior theory, defended by accounting literature, is infringing modern econometric concepts used in finance, in terms of identifying and correcting the non-stationarity of the temporal series. Therefore, a deductive methodology was used through indirect research. To reinforce the concepts and analyses carried out in the development of this research, case study data were used and processed through the econometric software E-views. Finally, it is concluded that the theory used in accounting literature about the use of statistical techniques in cost behavior does not consider the stationarity analysis of the series and that, therefore, it generates statistical inconsistencies (spurious regressions) in the forecasting of this referred behavior. Thus, this research is relevant in scientific evolution by opening a broader discussion in an incipient area of Accountancy, i.e. econometrics applied to accounting.

Keywords: Cost Accounting, Econometrics applied to Accounting, Stationarity 


\section{INTRODUÇÃO}

O contexto atual, em que se encontram inseridas empresas dos mais variados setores e segmentos, exige uma busca intensa pela melhoria da competitividade e postura empreendedora. As pressões ambientais, tanto em termos econômicos (lucros, remuneração do capital etc.), como sociais (empregos, eficiência dos recursos utilizados, preservação do meio ambiente etc.), fazem com que as empresas procurem cada fez mais inovar, seja no modo de fazer ou como melhor fazer.

Naturalmente isso retrata o grande avanço tecnológico observado nas últimas décadas, principalmente com o advento da robótica, que revolucionou o processo produtivo de muitas empresas.

Por outro lado, a tentativa de se administrar esse novo modelo de organização, centrada em planos estratégicos de atuação, tendo em vista as diversas variáveis internas e externas, remete ao antigo problema das empresas, principalmente as industriais, que é o custo de suas operações.

Isso tem levado as empresas a investirem em programas de qualidade e em otimização dos custos no processo produtivo, com o intuito de barganhar melhor competitividade. Com isso, estudos sobre o comportamento dos custos vêm tomando relevância não só nos meios acadêmicos, como também em áreas que lidam diretamente com as atividades empresariais (MEDEIROS, 2005).

Essa relevância está ligada ao fato de que muitas decisões gerenciais são baseadas na previsão de custos e no seu comportamento. No entanto, como indagam Shank e Govindarajam (1995): "qual a variável que melhor explica as mudanças no custo?" a primeira vista a "variação da produção ou atividade" seria a melhor resposta, todavia, como afirmam Garrison e Noreen (2001) "as tentativas de tomada de decisão sem o pleno conhecimento dos custos envolvidos - e de como eles podem variar em função do nível de atividades - pode levar ao desastre". Autores como Garrison e Noreen (2001); ludícibus (1989); Atkinson et al. (2000); Horngren et al. (2000); Maher (2001) Banker e Johnson (1993) trazem em suas obras diversas metodolo- gias para se estimar o comportamento dos custos. Uma das formas de análise comportamental dos custos é feita através da análise de Regressão, que utiliza dados históricos para estimar a função de custos.

Sabe-se, no entanto, que o estudo do comportamento dos custos fica ainda mais complexo quando se tenta prever o comportamento dos Custos Indiretos de Fabricação (CIF), pois esses possuem uma fraca característica de identificação com o volume de produção.

Os autores citados, anteriormente, propõem o estudo dessa categoria de custos através de regressões simples e múltiplas, utilizando diversas variáveis independentes relacionadas ao processo produtivo (horas-máquina, horas de mão-de-obra direta e/ou indireta, tempo de setup etc.), de forma a encontrar-se a melhor combinação de variáveis que explique o comportamento dos CIFs.

O uso desse tipo de técnica, segundo os autores, é mais consistente e facilita o entendimento do comportamento dos custos, formulando-se, portanto, uma teoria sobre essa temática.

$\mathrm{Na}$ contramão dos aspectos abordados, surge a teoria econométrica que levanta a possibilidade de alguns modelos econômicos terem um relacionamento espúrio, ou seja, o efeito estatístico significativo do modelo regressivo entre a variável dependente e a(s) variável(eis) independente(s) pode ser obra do acaso.

Diante desse impasse conceitual e teórico, a proposta deste trabalho é testar a teoria tradicional declarada por renomados autores, nacionais e internacionais, acerca da matéria, à luz da teoria econométrica, emergindo o seguinte questionamento: Estará a teoria da Análise Estatística do Comportamento dos Custos, defendida pela literatura contábil, ferindo conceitos modernos da Econometria utilizados em finanças?

Diante disso, proceder-se-á a um levantamento bibliográfico específico da matéria, além de um caso prático que reforce as conclusões retiradas neste artigo.

\section{CONCEITOS DE CUSTOS}

Para entender-se o comportamento dos custos, precisa-se antes entender suas categorias e características. Pode-se classificar custos por duas vertentes, segundo Garrison e Noreen (2001); Martins (2003); Hornegren, Foster e Datar (2000): uma pela apropriação ao objeto de custo e outra pelo comportamento em relação à variação do volume de produção. Entende-se como objeto de custo qualquer coisa - produto, linha de produto, cliente, departamento etc. - para a qual se deseje informação de custo (GARRISON e NOREEN, 2001). Neste trabalho, o objeto de custo será o produto ou produtos de uma organização, mas essa premissa não anula as considerações que serão evidenciadas neste artigo para os demais tipos de objetos de custos, apenas uma adoção intencional para facilitar o entendimento do trabalho. Nessa perspectiva, os custos podem ser classificados:

Quanto a sua Apropriação:

a) Custos Diretos: são aqueles que podem ser fácil e diretamente identificados ao objeto de custo em causa;

b) Custos Indiretos: são aqueles não podem ser facilmente identificados ao objeto de custo em causa.

Quanto à previsão de Comportamento:

a) Custos Variáveis: são aqueles cujo total varia na razão direta das alterações do nível de atividade; 
b) Custos Fixos: são aqueles cujo total permanece constante, independentemente das alterações no nível de atividade.

Esses conceitos, no entanto, não retratam a verdadeira complexidade dos custos, tendo em vista as suas diferentes possibilidades de comportamento. Sabe-se que uma com- preensão mais clara do processo de custeio tem uma relevância fundamental nas empresas, pois é uma importante fonte de informações para a tomada de decisões. Gestores que buscam um maior entendimento desses custos têm a condição de melhor entendê-los nas diversas situações operacionais e de planejamento.

\section{METODOLOGIA PARA PREVISÃO DO COMPORTAMENTO DOS CUSTOS}

O processo de decisão cada vez mais exige atitudes rápidas e precisas na gestão das empresas. E conhecer os custos e suas variações é fator primordial para que a alternativa de decisão escolhida ocorra da forma mais acertada possível, no intuito de se otimizar o resultado.

$\mathrm{Na}$ busca de retornos satisfatórios, que expliquem da melhor maneira como os padrões de comportamento de custo variam em função das modificações ocorridas com seus direcionadores, são utilizadas funções matemáticas que tentam expressar esses padrões de comportamento. De acordo com Horngren et al. (2000, p. 235), são considerados dois pressupostos quando da análise das funções de custo:

1. As variações nos custos totais de um objeto de custo são explicadas pelas variações de um único direcionador de custo.

2. O comportamento do custo é adequadamente descrito por uma função linear de custo do direcionador de custo, dentro da faixa de interesse. Função linear de custo é a função de custo para a qual, dentro da faixa de interesse, o gráfico dos custos totais, em contraposição a um único direcionador de custo, é uma linha reta.

A não linearidade nos padrões de comportamento dos custos, também, pode ocorrer, ou seja, há casos em que a relação entre o direcionador de custo e os custos totais não podem ser representados por uma reta.

O estabelecimento de uma função de custo de acordo com as premissas citadas anteriormente permitem a formulação de um modelo que possibilita determinar se existe relação de causa e efeito entre o direcionador de custo e os custos resultantes. Um conceito de modelo foi citado por Ackoff e Sasieni (1971), que diz:

Modelos são representações da realidade. Se fossem tão complexos e difíceis de controlar como a realidade, não haveria nenhuma vantagem em utilizá-los. Felizmente, podemos em geral construir modelos que são muito mais simples que a realidade e ainda assim conseguirmos empregá-los para prever e explicar fenômenos com alto grau de precisão. A razão disso é que, embora seja necessário um grande número de variáveis para prever um fenômeno com exatidão perfeita, um pequeno número de variáveis explica geralmente a maior parte dele. O truque, evidentemente, é achar as variáveis certas e a relação entre elas.
Vários autores renomados da área de custos, como Leone (2000), Hornegren, Foster e Datar (2000), Garrison e Noreen (2001), Maher (2001), ludícibus (1989), Datar (1993), Callado e Callado (2002), entre outros, sugerem a utilização da técnica de análise de regressão para a estimativa de comportamento dos custos, pois a técnica ajuda o administrador a determinar quão bem a equação estimada descreve a relação entre custo e direcionador. Ainda segundo os autores citados, o processo de regressão, também, permite a inclusão de mais de uma variável explicativa (regressão múltipla).

Várias associações e modelos podem ser construídos para facilitar a percepção do comportamento dos custos, em diversos níveis e categorias, ou seja, pode-se, por exemplo, querer identificar como se comportam os custos de mão-deobra direta, a fim de verificar suas características, se são variáveis, fixos ou mistos; ou ainda, estudar o comportamento dos Custos Indiretos, com a finalidade de entender melhor seu comportamento para auxiliar em decisões de encomenda, orçamento de custos, fixação de taxas predeterminadas de aplicação dos CIFs para rateio, etc.

ludícibus (1989), por exemplo, sugere que o analista de custos identifique direcionadores de custos potenciais através do conhecimento dos processos (plausibilidade econômica) que expliquem o comportamento dos CIFs. E que, dentre as diversas combinações de variáveis independentes (direcionadores), escolha o modelo estatisticamente mais significativo.

O conhecimento mais profundo das ferramentas estatísticas torna-se imprescindível nesse momento, pois, como se refere ludícibus (1989), a análise superficial dos parâmetros e testes estatísticos pode induzir ao erro por parte do analista de custos.

A grande maioria dos autores sugere a utilização de softwares estatísticos para auxiliar na construção desses modelos, pois, quando se parte para modelos de regressão múltipla, os cálculos matemáticos ficam mais complexos e extensos, além da grande quantidade de testes e parâmetros estatísticos que podem ser extraídos de uma regressão. Portanto, a fim de simplificar o entendimento do trabalho, será demonstrada uma análise de regressão simples envolvendo apenas duas variáveis (uma dependente e uma independente), extraídas do software E-views, que é um pacote estatístico muito utilizado e referenciado pela literatura Econométrica. 


\section{UM EXEMPLO PRÁTICO DE ANÁLISE DE REGRESSÃO EM CUSTOS}

Esse exemplo foi um caso demonstrado por Garrison e Noreen (2001), na empresa Ramon Company que produz uma ampla variedade de produtos, em diversas instalações. Em uma de suas unidades de componentes elétricos, teve dificuldade com as flutuações mensais dos custos indiretos de fabricação (CIF), que dificultaram a estimativa do nível de custos indiretos a ser incorrido em qualquer outro mês. O administrador da unidade desejava poder estimar os custos indiretos com precisão, a fim de melhor planejar suas atividades financeiras e operacionais. Um dos membros do quadro de contabilidade sugeriu determinar o comportamento dos custos indiretos, que, então, poderiam ser previstos a partir de um orçamento das horas de mão-de-obra direta, tendo em vista uma publicação da associação comercial que revelava tal correlação entre as variáveis no setor de atuação da empresa.

Diante disto, decidiu-se fazer um estudo histórico do comportamento dos custos indiretos, através da análise de regressão simples, em que os custos indiretos de fabricação seriam a variável dependente $(Y)$ e as horas de mão-deobra direta a variável independente $(X)$. Ou seja:

$$
Y(C I F)=\beta_{1}+\beta_{2} \cdot X(H M O D)+\varepsilon
$$

em que: $\beta_{1}$ e $\beta_{2}$ são os coeficientes que determinam o relacionamento linear entre as variáveis $\mathrm{Y}$ e $\mathrm{X} ; \varepsilon$ é a perturbação estocástica do modelo (Erro ou resíduo).

Inserindo os dados no software E-views e solicitando a análise de regressão, tem-se o resultado apresentado no Quadro 10 :

Inserindo os valores fornecidos pelo E-views na equação 1, o modelo de previsão do comportamento de custos indiretos de fabricação para o referido caso é:

$$
Y(C I F)=39.859,47+2,15488 \cdot X(H M O D)+\varepsilon
$$

Verifica-se, através do Quadro 1, que existe um forte relacionamento entre as variáveis. $O R^{2}$ ( $R$-squared) está alto, indicando que cerca de $91 \%$ do comportamento da variável Custos Indiretos de Fabricação é explicada pela variável independente Horas de Mão-de-obra. O teste ANOVA (F-statistic), utilizado para verificar a significância

Tabela 1 Dados históricos de horas de mão-de-obra direta e custos indiretos de fabricação da Ramon Company (1997-1998)

\begin{tabular}{l|c|c|c|c}
\multirow{2}{*}{ Mês } & \multicolumn{2}{|c|}{1997} & \multicolumn{2}{c}{1998} \\
\cline { 2 - 5 } & Horas de MOD & CIF (\$) & Horas de MOD & CIF (\$) \\
Janeiro & 20.000 & 84.000 & 21.000 & 86.000 \\
\hline Fevereiro & 25.000 & 99.000 & 24.000 & 93.000 \\
\hline Março & 22.000 & 89.500 & 23.000 & 93.000 \\
\hline Abril & 23.000 & 90.000 & 22.000 & 87.000 \\
\hline Maio & 20.000 & 81.500 & 20.000 & 80.000 \\
\hline Junho & 19.000 & 75.500 & 18.000 & 76.500 \\
\hline Julho & 14.000 & 70.500 & 12.000 & 67.500 \\
\hline Agosto & 10.000 & 64.500 & 13.000 & 71.000 \\
\hline Setembro & 12.000 & 69.000 & 15.000 & 73.500 \\
\hline Outubro & 17.000 & 75.000 & 17.000 & 72.500 \\
\hline Novembro & 16.000 & 71.500 & 15.000 & 71.000 \\
\hline Dezembro & 19.000 & 78.000 & 18.000 & 75.000
\end{tabular}

Fonte: Garrison e Noreen (2001, p. 159)

\begin{tabular}{|c|c|c|c|c|}
\hline \multicolumn{1}{|c|}{ Variable } & Coefficient & Std. Error & t-Statistic & Prob. \\
\hline C & 39859.47 & 2668.339 & 14.93793 & 0.0000 \\
\hline HMOD & 2.154880 & 0.143702 & 14.99548 & 0.0000 \\
\hline R-squared & 0.910882 & Mean dependent var & 78916.67 \\
\hline Adjusted R-squared & 0.906831 & S.D. dependent var & 9304.822 \\
\hline S.E. of regression & 2840.159 & Akaike info criterion & 18.82076 \\
\hline Sum squared resid & $1.77 \mathrm{E}+08$ & Schwarz criterion & 18.91893 \\
\hline Log likelihood & -223.8492 & F-statistic & 224.8645 \\
\hline Durbin-Watson stat & 1.225580 & Prob(F-statistic) & 0.000000 \\
\hline
\end{tabular}

Quadro 1 Output do E-views do resumo dos dados estatísticos (regressão simples) 
estatística do modelo, indica, no exemplo, que existe uma relação forte entre as variáveis a qualquer nível de significância, pois o "prob(F-statistic)" é zero.

O teste $\mathrm{t}$ ( $t$-statistic) dos coeficientes da regressão, também revelou uma inquestionável relação entre as variáveis, tendo em vista que o "prob." extraído foi zero, indicando que a hipótese de nulidade dos coeficientes estimados (interseção e Horas de Mão-de-Obra Direta) serem iguais a zero foi rejeitada, a qualquer nível de significância. Logo, pode-se concluir que a equação (ou modelo estimativo) extraída da análise de regressão tem um forte poder de relação explicativa entre as variáveis estudadas.

Outro parâmetro a ser observado quando se utilizam técnicas de regressão é a verificação da auto-correlação dos erros, tendo em vista que essa ocorrência torna a relação de baixa qualidade, ou seja, os seus coeficientes estarão enviesados provocando distorções nos desvios-padrões e no $\mathrm{R}^{2}$ (BROOKS, 2002). O teste oferecido pelo software utilizado no trabalho é o teste Durbin-Watson, que é um dos testes mais simples para a verificação da auto-correlação dos resíduos de uma regressão. Segundo ludícibus (1989) esse parâmetro deve estar em torno do numeral dois. No entanto, o referido autor não indica o quão distante desse valor (numeral 2) pode-se admitir a possibilidade da não auto-correlação dos erros. Contudo, a análise desse teste não é tão simples assim, mas como o objetivo deste trabalho não é discorrer sobre todas as técnicas e premissas das relações regressivas, será explanado de forma didática, deixando de lado o rigor e a forma matemática de sua concepção, acreditando que a desconsideração desse tipo de teste nas análises estatísticas afetará a qualidade da regressão.

Deve-se utilizar a tabela estatística Durbin-Watson, presente em vários livros de Econometria e Estatística, cuja consulta é muito simples. Como se sabe, uma tabela é formada por linhas e colunas e o parâmetro a ser consultado como linha, neste caso, é o número de observações da regressão (T), que no caso em tela são 24 meses, e a coluna do número de variáveis explicativas (sem considerar a constante), que no caso é apenas 1 (Horas de Mão-de-Obra Direta). Os valores críticos da tabela para essa situação $(24,1)$ são $d_{L}=1,04$ e $d_{u}=1,20$. Isso, graficamente, indica o seguinte:
Agora, pode-se concluir, de acordo com as premissas do teste Durbin-Watson, que os resíduos desse modelo não estão auto-correlacionados e, portanto, todas as análises feitas a respeito dos seus coeficientes, Erro Padrão e grau de determinação $\left(R^{2}\right)$ são válidas.

Deve-se, nesse tipo de análise, apesar de ser ignorado em quase todas as obras de contabilidade acerca do assunto, verificar o Erro Padrão da estimativa, de forma a determinar o grau de distorção provocada no modelo. Por exemplo, se o gestor da unidade administrativa estudada, a empresa Ramon Company, tivesse estimado em seu orçamento um gasto de 25.000 horas de Mão-de-Obra Direta para o mês de Janeiro de 1999, ele teria a seguinte previsão de Custos Indiretos de Fabricação (CIF), utilizando a equação 2:

$$
Y(C I F)=39.859,47+(2,15488 \times 25.000) \approx 93.731,47
$$

Através das premissas da Teoria do Limite Central esse resultado indica que existe uma probabilidade de $68,26 \%$ dos Custos Indiretos de Fabricação estarem no intervalo de (93.731,47 - Erro Padrão) e (93.731,47 + Erro Padrão) [(IUDÍCIBUS, 1989); (STEVESON, 1981)], ou seja, utilizando-se o valor do erro padrão (S.E. of regression) dado pelo software E-views, no Gráfico 20 , observa-se o seguinte.

Conforme o Gráfico 2, para uma previsão de 25.000 Horas de Mão-de-Obra Direta, o valor esperado (médio) de Custos Indiretos de Fabricação é de \$93.731,47, com uma probabilidade de que $68,26 \%$ desse valor esteja entre \$ $90.891,31$ e $\$ 96.571,63$. Se aumentar mais um Erro Padrão para cada lado, a probabilidade aumentará para $95,44 \%$; mais dois Erros Padrões, a probabilidade aumentará para 99,72\%. Quanto maior for a quantidade de Erros Padrões atribuídos ao intervalo, mais próximo de $100 \%$ chegará a probabilidade, no entanto, nunca chegará a 100\%, pois se trata de um estudo aleatório de probabilidades e não determinístico [(STEVENSON, 1981); (IUDÍCIBUS, 1989)].

Contudo, quanto maior o Erro Padrão (S.E. of regression) maior a possibilidade de o valor estimado ser diferente do real, ou seja, diminuindo o nível de precisão da estimativa.

Todavia, as premissas estudadas até esse momento não tornam os modelos infalíveis, apenas os tornam es-

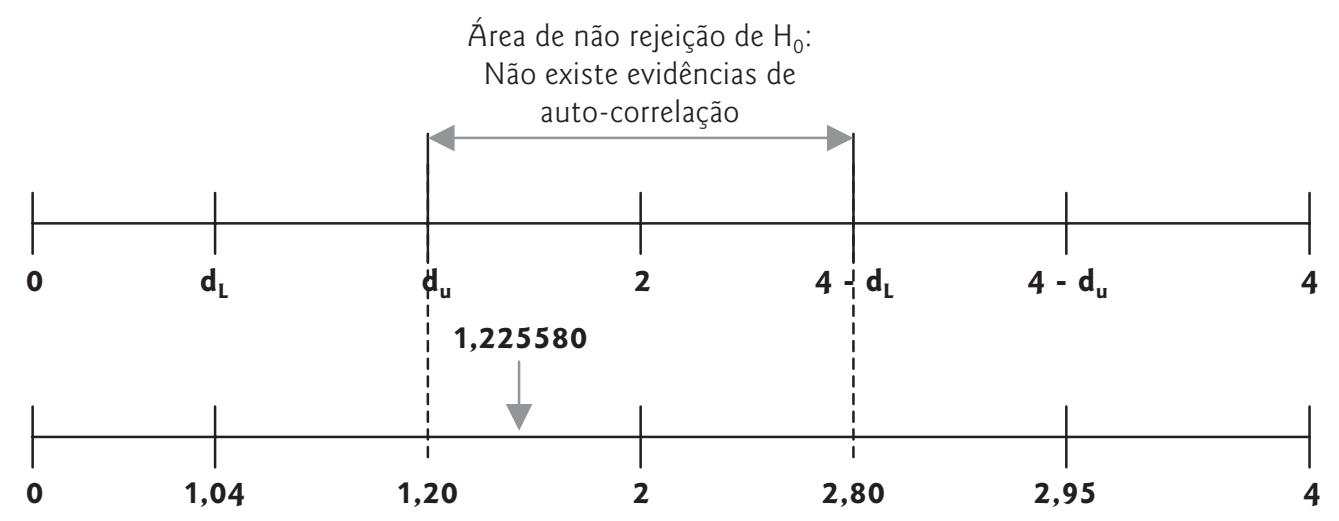

Gráfico 1 Análise do teste Durbin-Watson para auto-correlação dos resíduos 


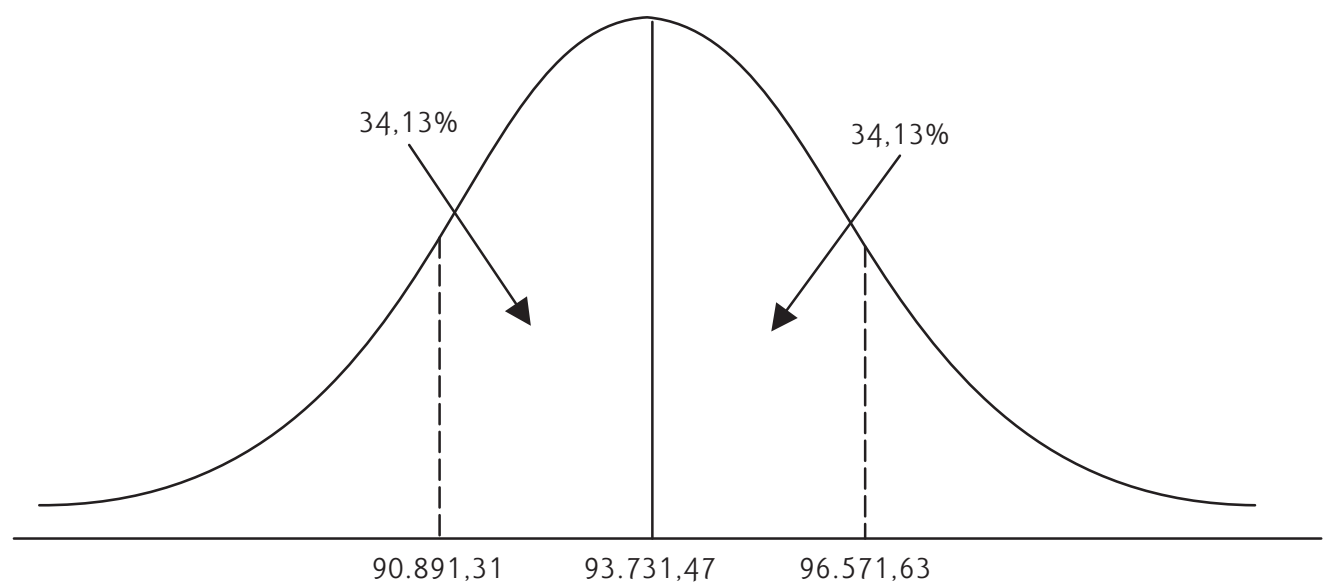

Gráfico 2 Distribuição de probabilidade (curva normal) da estimativa

tatisticamente (previsão) mais robustos ou significativos. Outros aspectos, porém, devem ser considerados além dos já vistos, quando se trata de séries temporais em Finanças (incluindo-se, nesse, campo a Contabilidade). Estudos recentes na área indicam que as análises estatísticas clássicas feitas sobre resultados de uma regressão podem ser faIhos, tendo em vista a possibilidade dessa relação ser obra do acaso. Por exemplo, segundo Karmel e Polasek (1981), foi feita uma regressão entre o número de nascimento de bebês e o número de surgimento de cegonhas em uma determinada região, e foi observada uma relação estatisticamente forte, mas como se sabe, em termos de uma realidade, totalmente descompassada. Os autores chamam esse tipo de relação de regressões espúrias, ocasionadas pela não estacionariedade (tendência aleatória ou estocástica) da série temporal e é esse tema que será discutido nos tópicos a seguir.

\section{TEORIA ECONOMÉTRICA: A EXISTÊNCIA DE RAİZES UNITÁRIAS EM SÉRIES TEMPORAIS}

Segundo Fava e Cati (1995), a origem da discussão de raiz unitária nas séries econômicas e financeiras está no debate sobre a estacionariedade ou não da tendência, sendo que grande parte dos dados utilizados na análise empírica em economia e finanças é em forma de uma série temporal.

Uma série com uma tendência estocástica se diferencia de outra com uma tendência determinística, pois as mudanças na mesma deixam de ter um caráter transitório e passam a apresentar um caráter permanente [(GUJARATI, 2000); (HILL, GRIFFITHS e JUDGE, 1999)].

A presença de uma tendência estocástica implica que flutuações em uma série temporal são o resultado de choques não somente no componente transitório ou cíclico, mas também no componente de tendência. (GUJARATI, 2000)

Logo, a determinação da presença de raiz unitária é relevante para a Economia, pois auxilia no processo de verificação de várias teorias. Vale ressaltar que, segundo Brooks (2002), esse tipo de estudo se aplica a Finanças e, desta forma, as variáveis financeiras (Contabilidade).

A utilização dos modelos de regressão envolvendo séries temporais não estacionárias pode conduzir ao problema que se convencionou chamar de regressão espúria [(GUJARATI, 2000); (BROOKS, 2002); (HILL, GRIFFITHS e JUDGE, 1999); (MEDEIROS, 2005)], ou seja, quando uma regressão tem um grau de determinação $\left(\mathrm{R}^{2}\right)$ muito alto e parâmetros estatísticos significativos (teste $\mathrm{F}$ e t), mas sem uma relação "realística" entre as variáveis (HARRIS, 1995). Se uma variável em um modelo de regressão não for estacionária, então, as análises sobre a significância dos parâmetros ou coeficientes da regressão podem ser não válidas (BROOKS, 2002). Isso ocorre devido ao fato de que a presença de uma tendência, decrescente ou crescente, em ambas as séries leva a um alto valor do $\mathrm{R}^{2}$, mas não necessariamente, a presença de uma relação verdadeira entre séries (GUJARATI, 2000).

Brooks (2002) retrata uma pesquisa feita com 1.000 regressões com várias variáveis não estacionárias totalmente independentes e constatou-se que grande parte das combinações deu um $\mathrm{R}^{2}$ muito alto e a estatística t e F significantes. Isto é, a presença de raiz unitária na série temporal conduz a resultados viesados, invalidando os pressupostos da estatística clássica de que a média e a variância são constantes ao longo do tempo e, com isso, mascarando o relacionamento entre duas ou mais variáveis (BROOKS, 2002).

Portanto, um processo estocástico estacionário é aquele cuja média e variância são constantes ao longo do tempo e o valor da covariância entre dois períodos de tempo (defasagem) depende apenas da distância temporal entre eles e não do período de tempo efetivo em que a covariância é calculada - Processo Estocástico Fracamente Estacionário (GUJARATI, 2000). 
Ou seja, para que uma série temporal estocástica qualquer $\left(\mathrm{Y}_{\mathrm{t}}\right)$ seja considerada estacionária, deverá ter as seguintes propriedades, segundo Gujarati (2000):

Média: $E\left(Y_{i}\right)=\mu$

Variância: $\operatorname{var}\left(Y_{i}\right)=E\left(Y_{i}-\mu\right)^{2}=\sigma^{2}$

Covariância: $\operatorname{cov}\left(Y_{t}, Y_{t+k}\right)=E\left[\left(Y_{t}-\mu\right) \cdot\left(Y_{t+k}-\mu\right)\right]$

Para se detectar a presença de raiz unitária em uma séria temporal existem alguns testes, os mais conhecidos são: teste DF (Dickey-Fuller) e o ADF (Augmented DickeyFuller ou Dickey-Fuller Aumentado).

O objetivo básico do primeiro (DF) é fazer um teste de hipótese sobre aleatoriedade da defasagem.

$$
\Delta Y_{t}=Y_{t}-Y_{t-1}=u_{t}
$$

Isto é,

$$
Y_{t}=\phi \cdot Y_{t-1}+u_{t}
$$

Se o coeficiente do modelo auto-regressivo de ordem 1 $(A R(1)=$ equação 7$)$ for igual a 1 , então a variável estocástica $y_{t}$ tem uma raiz unitária, logo:

$H_{0}: \phi=1$, a série contém raiz unitária.

$H_{1}: \phi<1$, a série não contém raiz unitária.

Usualmente, segundo Brooks (2002), utiliza-se a seguinte sentença para a realização do referido teste:

Atribui-se $y_{t-1}$ nos dois lados da equação 7 :

$$
\begin{gathered}
Y_{t}-Y_{t-1}=\phi \cdot\left(Y_{t-1}-Y_{t-1}\right)+\varepsilon_{t} \\
\therefore \Delta Y_{t}=(\phi-1) \cdot Y_{t-1}+\varepsilon_{t}
\end{gathered}
$$

Se $\psi=(\phi-1), \log 0:$

$$
\Delta Y_{t}=\psi \cdot Y_{t-1}+u_{t}
$$

Então o teste de hipótese $\phi=1$, da equação 7 , é equivalente ao teste $\psi=0$, da equação 9 , em que: $\mathrm{H}_{0}: \psi=0$, a série contém raiz unitária e $\mathrm{H}_{1}: \psi<0$, a série não contém raiz unitária. como:

Logo, o teste estatístico Dickey-Fuller (DF) é definido

$$
\text { Teste Estatístico }(D F)=\frac{\psi}{\text { Desvio }-\operatorname{Padrão}(\psi)}
$$

O teste estatístico não segue uma distribuição $t$ usual, os valores críticos derivam dos experimentos de Monte Carlo, que podem ser encontrados em alguns livro de econometria recente.

Já o teste ADF (Augmented Dickey-Fuller ou Dickey-Fuller aumentado), argumenta que o exposto acima não considera a possibilidade de uma auto-correlação dos erros ( $\left.u_{\mathrm{f}}\right)$, com isso quebra-se uma das hipóteses econométricas. No entanto, o teste referido corrige esse erro.

No trabalho será utilizado o software E-Views para realizar os dois testes supramencionados.

Todavia, uma vez detectada a presença do efeito não estacionário na série histórica, deve-se diferenciar as variáveis, ou seja, utilizar no modelo regressivo a diferença entre o valor da série e o seu valor temporal defasado, para todas as observações, na mesma proporção do número de raízes unitárias, pois, assim, elementos de longo prazo entre as variáveis são eliminados. Tomando, como exemplo, um modelo regressivo genérico que tenha uma raíz unitária:

$$
Y_{t}=\alpha+\beta \cdot X_{t}+\varepsilon_{t}
$$

Logo, corrige-se a estacionariedade da equação 10 pela diferenciação única de cada uma de suas variáveis:

$$
\begin{gathered}
Y_{t}-Y_{t-1}=\alpha+\beta \cdot\left(X_{t}-X_{t-1}\right)+\varepsilon_{t} \\
\therefore \Delta Y_{t}=\alpha+\beta \cdot \Delta X_{t}+\varepsilon_{t}
\end{gathered}
$$

Entretanto, como demonstra a equação 11 , as variáveis do modelo não mais serão os valores históricos absolutos da série e sim seus valores diferenciados. Isso quer dizer que, uma vez detectada a estacionariedade da série, a análise da qualidade ( $\mathrm{R}^{2}$, teste $\mathrm{F}$, teste $\mathrm{t}$, Durbin-Watson etc.) do modelo deve ser feita sobre a equação 11 e não mais sobre a equação 10. Se for verificado, após a diferenciação, que a qualidade estimativa do modelo é fraca, ele deve ser abandonado.

\section{APLICAÇÃO DO TESTE}

Retomando o exemplo prático da Ramon Company (GARRISON E NOREEN, 2001), em que, como já foi visto, o analista de custos se propõe a estimação dos Custos Indiretos de Fabricação (CIF) através do direcionador Horas de Mão-de-Obra Direta.

Será testada, todavia, a estacionariedade das variáveis, tanto a independente como a dependente. Se ambas ou apenas uma delas contiver raízes unitárias (mesmo que a outra não tenha), deve-se diferenciar todas as variáveis envolvidas no modelo e rodar novamente regressão.

Através do Quadro $2 \theta$, verifica-se que a variável CIF tem pelo menos 1 raiz unitária, pois o valor do teste ADF está na zona de não rejeição da hipótese nula em todos os níveis usuais de significância (Valores críticos a $1 \%$ e $5 \%$ ), já que a hipótese nula é que existe uma raiz unitária. 


\begin{tabular}{|c|c|c|c|c|}
\hline ADF Test Statistic & -2.869330 & \multicolumn{2}{|c|}{$1 \%$ Critical Value* } & -3.7667 \\
\hline & & \multicolumn{2}{|c|}{$5 \%$ Critical value } & -3.0038 \\
\hline & & \multicolumn{2}{|c|}{$10 \%$ Critical Value } & -2.6417 \\
\hline \multicolumn{5}{|c|}{ *MacKinnon critical values for rejection of hypothesis of a unit root. } \\
\hline \multicolumn{5}{|c|}{ Augmented Dickey-Fuller Test Equation } \\
\hline \multicolumn{5}{|c|}{ Dependent Variable: $D(C I F)$} \\
\hline \multicolumn{5}{|c|}{ Method: Least Squares } \\
\hline \multicolumn{5}{|c|}{ Date: 10/28/05 Time: 21:33 } \\
\hline \multicolumn{5}{|c|}{ Sample(adjusted): 1997:03 1998:12 } \\
\hline \multicolumn{5}{|c|}{ Included observations: 22 after adjusting endpoints } \\
\hline Variable & Coefficient & Std. Error & t-Statistic & Prob. \\
\hline CIF(-1) & -0.344018 & 0.119895 & -2.869330 & 0.0098 \\
\hline$D(C I F(-1))$ & 0.266632 & 0.176405 & 1.511480 & 0.1471 \\
\hline$C$ & 26197.18 & 9551.397 & 2.742759 & 0.0129 \\
\hline$R$-squared & 0.309284 & \multicolumn{2}{|c|}{ Mean dependent var } & -1090.909 \\
\hline Adjusted R-squared & 0.236577 & \multicolumn{2}{|c|}{ S.D. dependent var } & 5662.400 \\
\hline S.E. of regression & 4947.469 & \multicolumn{2}{|c|}{ Akaike info criterion } & 19.97726 \\
\hline Sum squared resid & $4.65 \mathrm{E}+08$ & \multicolumn{2}{|c|}{ Schwarz criterion } & 20.12604 \\
\hline Log likelihood & -216.7499 & \multicolumn{2}{|l|}{ F-statistic } & 4.253846 \\
\hline Durbin-Watson stat & 1.828291 & \multicolumn{2}{|c|}{ Prob(F-statistic) } & 0.029740 \\
\hline
\end{tabular}

Quadro 2 Output do E-Views do teste ADF para variável (CIF)

Para saber se a série temporal tem mais de uma raiz unitária deve rodar outra vez o teste, só que, agora, com a diferenciação, se for rejeitada a hipótese nula nessa situação, isso indica que a série temporal tem apenas uma raiz unitária. Se não for rejeitada, deve-se diferenciar a $2^{\text {a }}$ vez e assim por diante, até ser rejeitada a hipótese nula.

Verifica-se, através do Quadro $3 \mathbf{0}$, que mesmo com a primeira diferenciação a tendência permanece estacionária, pois não foi rejeitada a hipótese nula de existência da raiz unitária. Portanto, deve-se diferenciar outra vez.

Com o resultado do Quadro $4 \boldsymbol{\bullet}$, pode-se afirmar que a série temporal (CIFs) tem duas raízes unitárias, já que na $2^{\circ}$ diferenciação a hipótese nula foi, finalmente, rejeitada ao nível de $5 \%$ de significância. Deve-se fazer como já dito, o mesmo teste para todas as variáveis, portanto, o teste para a variável Horas de Mão-de-obra Direta foi o apresentado no Quadro 50.

\begin{tabular}{|c|c|c|c|c|}
\hline ADF Test Statistic & -2.145948 & \multicolumn{2}{|c|}{$1 \%$ Critical Value* } & -3.7856 \\
\hline & & \multicolumn{2}{|c|}{$5 \%$ Critical Value } & -3.0114 \\
\hline & & \multicolumn{2}{|c|}{$10 \%$ Critical Value } & -2.6457 \\
\hline \multicolumn{5}{|c|}{ *MacKinnon critical values for rejection of hypothesis of a unit root. } \\
\hline \multicolumn{5}{|c|}{ Augmented Dickey-Fuller Test Equation } \\
\hline \multicolumn{5}{|c|}{ Dependent Variable: $D(C I F, 2)$} \\
\hline \multicolumn{5}{|c|}{ Method: Least Squares } \\
\hline \multicolumn{5}{|c|}{ Date: 10/28/05 Time: $21: 35$} \\
\hline \multicolumn{5}{|c|}{ Sample(adjusted): 1997:04 1998:12 } \\
\hline \multicolumn{5}{|c|}{ Included observations: 21 after adjusting endpoints } \\
\hline Variable & Coefficient & Std. Error & t-Statistic & Prob. \\
\hline$D(C I F(-1))$ & -0.562552 & 0.262146 & -2.145948 & 0.0458 \\
\hline$D(C I F(-1), 2)$ & -0.119783 & 0.179330 & -0.667949 & 0.5126 \\
\hline$C$ & -201.3272 & 1202.183 & -0.167468 & 0.8689 \\
\hline$R$-squared & 0.368363 & \multicolumn{2}{|c|}{ Mean dependent var } & 642.8571 \\
\hline Adjusted R-squared & 0.298181 & \multicolumn{2}{|c|}{ S.D. dependent var } & 6383.852 \\
\hline S.E. of regression & 5348.048 & \multicolumn{2}{|c|}{ Akaike info criterion } & 20.13841 \\
\hline Sum squared resid & $5.15 E+08$ & \multicolumn{2}{|c|}{ Schwarz criterion } & 20.28763 \\
\hline Log likelihood & -208.4534 & \multicolumn{2}{|l|}{ F-statistic } & 5.248696 \\
\hline Durbin-Watson stat & 1.783614 & \multicolumn{2}{|c|}{ Prob(F-statistic) } & 0.016003 \\
\hline
\end{tabular}

Quadro 3 Output do E-Views do teste ADF para primeira diferença da variável (CIF) 


\begin{tabular}{|c|c|c|c|c|}
\hline ADF Test Statistic & -3.703724 & \multicolumn{2}{|c|}{$1 \%$ Critical Value* } & -3.8067 \\
\hline & & \multicolumn{2}{|c|}{$5 \%$ Critical Value } & -3.0199 \\
\hline & & \multicolumn{2}{|c|}{$10 \%$ Critical Value } & -2.6502 \\
\hline \multicolumn{5}{|c|}{ *MacKinnon critical values for rejection of hypothesis of a unit root. } \\
\hline \multicolumn{5}{|c|}{ Augmented Dickey-Fuller Test Equation } \\
\hline \multicolumn{5}{|c|}{ Dependent Variable: $D(C I F, 3)$} \\
\hline \multicolumn{5}{|l|}{ Method: Least Squares } \\
\hline \multicolumn{5}{|c|}{ Date: 10/28/05 Time: 21:36 } \\
\hline \multicolumn{5}{|c|}{ Sample(adjusted): 1997:05 1998:12 } \\
\hline \multicolumn{5}{|c|}{ Included observations: 20 after adjusting endpoints } \\
\hline Variable & Coefficient & Std. Error & t-Statistic & Prob. \\
\hline$D(C I F(-1), 2)$ & -1.378227 & 0.372119 & -3.703724 & 0.0018 \\
\hline$D(C I F(-1), 3)$ & 0.038859 & 0.186725 & 0.208109 & 0.8376 \\
\hline$C$ & 279.6596 & 1381.656 & 0.202409 & 0.8420 \\
\hline$R$-squared & 0.680557 & \multicolumn{2}{|c|}{ Mean dependent var } & -225.0000 \\
\hline Adjusted R-squared & 0.642975 & \multicolumn{2}{|c|}{ S.D. dependent var } & 10291.12 \\
\hline S.E. of regression & 6149.104 & \multicolumn{2}{|c|}{ Akaike info criterion } & 20.42348 \\
\hline Sum squared resid & $6.43 \mathrm{E}+08$ & \multicolumn{2}{|c|}{ Schwarz criterion } & 20.57284 \\
\hline Log likelihood & -201.2348 & \multicolumn{2}{|l|}{ F-statistic } & 18.10882 \\
\hline Durbin-Watson stat & 1.852368 & \multicolumn{2}{|c|}{ Prob(F-statistic) } & 0.000061 \\
\hline
\end{tabular}

Quadro 4 Output do E-Views do teste ADF para segunda diferença da variável (CIF)

Constatou-se, através do Quadro $5 \mathbf{0}$, que a variável HMOD, também, tem duas raízes unitárias, que, portanto, o modelo estimativo dos Custos Indiretos de Fabricação deve sofrer duas diferenciações para corrigir os efeitos da estacionariedade sobre a equação. Logo,

$$
\Delta(\Delta Y(C I F))=\alpha+\beta \cdot \Delta(\Delta X(H M O D))+\varepsilon
$$

Feito isso, deve-se rodar novamente a regressão (através da equação 12) e analisar os resultados estatísticos da nova estimação apresentada no Quadro 60.

Observando os novos dados estatísticos da nova regressão, através do Quadro 6, considerando as diferenciações das variáveis, verifica-se uma baixa no seu poder explicativo, já que o $\mathrm{R}^{2}$ caiu para aproximadamente $73 \%$

\begin{tabular}{|c|c|c|c|c|}
\hline ADF Test Statistic & -3.840050 & \multicolumn{2}{|c|}{$1 \%$ Critical Value* } & -3.8067 \\
\hline & & \multicolumn{2}{|c|}{$5 \%$ Critical value } & -3.0199 \\
\hline & & \multicolumn{2}{|c|}{$10 \%$ Critical Value } & -2.6502 \\
\hline \multicolumn{5}{|c|}{ *MacKinnon critical values for rejection of hypothesis of a unit root. } \\
\hline \multicolumn{5}{|c|}{ Augmented Dickey-Fuller Test Equation } \\
\hline \multicolumn{5}{|c|}{ Dependent Variable: $D(H M O D, 3)$} \\
\hline \multicolumn{5}{|l|}{ Method: Least Squares } \\
\hline \multicolumn{5}{|c|}{ Date: 10/28/05 Time: 21:39 } \\
\hline \multicolumn{5}{|c|}{ Sample(adjusted): 1997:05 1998:12 } \\
\hline \multicolumn{5}{|c|}{ Included observations: 20 after adjusting endpoints } \\
\hline Variable & Coefficient & Std. Error & t-Statistic & Prob. \\
\hline$D(H M O D(-1), 2)$ & -1.538625 & 0.400678 & -3.840050 & 0.0013 \\
\hline$D(H M O D(-1), 3)$ & 0.084700 & 0.227095 & 0.372972 & 0.7138 \\
\hline$C$ & 109.9912 & 795.5464 & 0.138259 & 0.8917 \\
\hline$R$-squared & 0.701149 & \multicolumn{2}{|c|}{ Mean dependent var } & 50.00000 \\
\hline Adjusted R-squared & 0.665990 & \multicolumn{2}{|c|}{ S.D. dependent var } & 6151.380 \\
\hline S.E. of regression & 3555.102 & \multicolumn{2}{|c|}{ Akaike info criterion } & 19.32764 \\
\hline Sum squared resid & $2.15 \mathrm{E}+08$ & \multicolumn{2}{|c|}{ Schwarz criterion } & 19.47700 \\
\hline Log likelihood & -190.2764 & \multicolumn{2}{|l|}{ F-statistic } & 19.94230 \\
\hline Durbin-Watson stat & 1.963917 & \multicolumn{2}{|c|}{ Prob(F-statistic) } & 0.000035 \\
\hline
\end{tabular}

Quadro 5 Output do E-Views do teste ADF para segunda diferença da variável (HMOD) 


\begin{tabular}{|c|c|c|c|c|}
\hline Variable & Coefficient & Std. Error & t-Statistic & Prob. \\
\hline$C$ & -339.4864 & 903.6568 & -0.375681 & 0.7111 \\
\hline$D(D(H M O D))$ & 1.765650 & 0.229866 & 7.681203 & 0.0000 \\
\hline R-squared & 0.746838 & Mean dependent var & -500.0000 \\
\hline Adjusted R-squared & 0.734180 & S.D. dependent var & 8218.736 \\
\hline S.E. of regression & 4237.393 & Akaike info criterion & 19.62779 \\
\hline Sum squared resid & $3.59 \mathrm{E}+08$ & Schwarz criterion & 19.72698 \\
\hline Log likelihood & -213.9057 & F-statistic & 59.00087 \\
\hline Durbin-Watson stat & 2.222867 & Prob(F-statistic) & 0.000000 \\
\hline
\end{tabular}

Quadro 6 Output do E-views do resumo dos dados estatísticos (nova regressão simples)

(antes era 91\%). Por outro lado, os testes " $\mathrm{t}$ " e " $\mathrm{F}$ " indicaram que o relacionamento entre as variáveis permanece ainda bastante significativo, portanto válido.

Levando em consideração que foi estimado, pelo gestor, 25.000 Horas de Mão-de-Obra Direta para janeiro de 1999, tem-se como segunda diferenciação o valor de 4.000 horas (conforme a Tabela 2 O). Incluindo esse valor na equação 12 de regressão tem-se o seguinte resultado:

$\Delta(\Delta Y(C I F))=-339,4864+1,765650 \cdot \Delta(\Delta X(H M O D))$
Logo,

$\Delta(\Delta Y(C I F))=-339,4864+(1,765650 \cdot 4.000) \approx 6.723$

Fazendo a diferenciação da variável dependente, Custos Indiretos de Fabricação, tem-se o resultado apresentado na Tabela 30.

Com os valores da Tabela 3, chega-se a uma previsão de Custos Indiretos de Fabricação, para dezembro de 1999 , de $\$ 85.723,00(75.000+4.000+6.723)$, ao invés dos $\$ 93.731,47$ do modelo anterior, que não considerava os efeitos da não estacionariedade da tendência.

Tabela 2 | Diferenciação na série temporal da variável HMOD (1997-1998)

\begin{tabular}{l|c|c|c}
\multicolumn{4}{c}{ Mplicação da Diferenciação na Série Temporal (1997- 1998) } \\
\hline Janeiro/97 & Horas de MOD (X) & $\mathbf{X}_{\mathbf{t}}-\mathbf{X}_{\mathbf{t}-\mathbf{1}} \mathbf{( Z )}$ & $\mathbf{Z}_{\mathbf{t}}-\mathbf{Z}_{\mathbf{t}-1}$ \\
\hline Fevereiro/97 & 20.000 & - & - \\
\hline Março/97 & 25.000 & 5.000 & - \\
\hline Abril/97 & 22.000 & -3.000 & -8.000 \\
\hline Maio/97 & 23.000 & 1.000 & 4.000 \\
\hline Junho/97 & 20.000 & -3.000 & -4.000 \\
\hline Julho/97 & 19.000 & -1.000 & 2.000 \\
\hline Agosto/97 & 14.000 & -5.000 & -4.000 \\
\hline Setembro/97 & 10.000 & -4.000 & 1.000 \\
\hline Outubro/97 & 12.000 & 2.000 & 6.000 \\
\hline Novembro/97 & 17.000 & 5.000 & 3.000 \\
\hline Dezembro/97 & 16.000 & -1.000 & -6.000 \\
\hline Janeiro/98 & 19.000 & 3.000 & 4.000 \\
\hline Fevereiro/98 & 21.000 & 2.000 & -1.000 \\
\hline Março/98 & 24.000 & 3.000 & 1.000 \\
\hline Abril/98 & 23.000 & -1.000 & -4.000 \\
\hline Maio/98 & 22.000 & -1.000 & 0 \\
\hline Junho/98 & 20.000 & -2.000 & -1.000 \\
\hline Julho/98 & 18.000 & -2.000 & 0 \\
\hline Agosto/98 & 12.000 & -6.000 & -4.000 \\
\hline Setembro/98 & 13.000 & 1.000 & 7.000 \\
\hline Outubro/98 & 15.000 & 2.000 & 1.000 \\
\hline Novembro/98 & 17.000 & 2.000 & 0 \\
\hline Dezembro/98 & 15.000 & -2.000 & -4.000 \\
\hline Previsão Janeiro/1999 & 25.000 & 5.000 \\
\hline & & & 4.000 \\
\hline
\end{tabular}


Tabela 3 | Diferenciação na série temporal da variável CIF (1997-1998)

\begin{tabular}{l|c|c|c}
\multicolumn{4}{c}{ Aplicação da Diferenciação na Série Temporal (1997- 1998) } \\
\hline \multicolumn{1}{c}{ Mês } & CIF (\$) & $\mathbf{Y}_{\mathbf{t}}-\mathbf{Y}_{\mathbf{t}-\mathbf{1}} \mathbf{( W )}$ & $\mathbf{W}_{\mathbf{t}}-\mathbf{W}_{\mathbf{t}-\mathbf{1}}$ \\
\hline Janeiro/97 & 84.000 & - & - \\
\hline Fevereiro/97 & 99.000 & 15.000 & - \\
\hline Março/97 & 89.500 & -9.500 & -24.500 \\
\hline Abril/97 & 90.000 & 500 & 10.000 \\
\hline Maio/97 & 81.500 & -8.500 & -9.000 \\
\hline Junho/97 & 75.500 & -6.000 & 2.500 \\
\hline Julho/97 & 70.500 & -5.000 & 1.000 \\
\hline Agosto/97 & 64.500 & -6.000 & -1.000 \\
\hline Setembro/97 & 69.000 & 4.500 & 10.500 \\
\hline Outubro/97 & 75.000 & 6.000 & 1.500 \\
\hline Novembro/97 & 71.500 & -3.500 & -9.500 \\
\hline Dezembro/97 & 78.000 & 6.500 & 10.000 \\
\hline Janeiro/98 & 86.000 & 8.000 & 1.500 \\
\hline Fevereiro/98 & 93.000 & 7.000 & -1.000 \\
\hline Março/98 & 93.000 & & -7.000 \\
\hline Abril/98 & 87.000 & -6.000 & -6.000 \\
\hline Maio/98 & 80.000 & -7.000 & -1.000 \\
\hline Junho/98 & 76.500 & -3.500 & 3.500 \\
\hline Julho/98 & 67.500 & -9.000 & -5.500 \\
\hline Agosto/98 & 71.000 & 3.500 & 12.500 \\
\hline Setembro/98 & 73.500 & 2.500 & -1.000 \\
\hline Outubro/98 & 72.500 & -1.000 & -3.500 \\
\hline Novembro/98 & 71.000 & -1.500 & -500 \\
\hline Dezembro/98 & 75.000 & 4.000 & 5.500 \\
\hline Previsão Janeiro/1999 & 85.723 & 10.723 & 6.723 \\
\hline
\end{tabular}

\section{CONCLUSÃO}

Primeiramente, é preciso ressaltar que o exemplo dado neste trabalho contém algumas limitações em termos de precisão dos conceitos discutidos, pois existe uma forte discussão no meio acadêmico sobre o tamanho mínimo da amostra a ser utilizada em técnicas de regressão e identificação da raiz unitária. No entanto, a maioria dos autores da área acredita que uma amostra de 30 observações (Observando os preceitos da Teoria do Limite Central) já tenderia a uma distribuição normal o que, portanto, possibilita sua utilização em análises dessa magnitude. Contudo, o objetivo central do estudo foi demonstrar a incoerência da teoria largamente encontrada na literatura contábil concernente ao assunto, não havendo, então, uma preocupação maior com a normalidade dos dados, tendo em vista que o intuito principal era a familiarização da técnica e utilização do ferramental estatístico para os profissionais de Contabilidade e afins.

Verificou-se que existe a necessidade de se estenderem as análises de forma a melhorar o poder de estimação dos modelos econométricos em contabilidade (Contabilometria), tendo em vista que o trabalho demonstrou a incon- sistência contida nas técnicas e práticas de utilização de técnicas de regressão com séries temporais financeiras (ou Contábeis).

Este estudo está inclinado à nova concepção da contabilidade moderna, no intuito de gerar valor aos seus usuários. Com isso, é imprescindível o estreitamento da Contabilidade com outras ciências, como a Economia, a Administração e a Estatística. Não se pode esquecer que essas técnicas são auxiliares ao processo decisório e não determinantes incondicionais do processo comportamental da decisão. Não se pode, também, deixar de lado a sensibilidade e a experiência de quem está lidando com esses tipos de dados, principalmente em Contabilidade.

Contudo, o pano de fundo da investigação em tela é propor uma ampliação da discussão no meio acadêmico e profissional no concernente ao estudo do comportamento dos custos (e de outras variáveis contábeis) utilizado em fins gerenciais ou não, ou seja, pesquisas posteriores, na forma de estudo de casos mais robustos, tornaram a teoria gerada neste trabalho mais forte. 


\section{Referências Bibliográficas}

ACKOFF, Russell Lincoln; SASIENI, Maurice W.. Pesquisa operacional. Rio de Janeiro: Livros Técnicos e Científicos, 1971.

ATKINSON, Anthony A. et al. Contabilidade Gerencial. São Paulo: Atlas, 2000.

BANKER, Rajiv D.; JOHNSON, Holly H.. An Empirical Study of Cost Drivers in the U.S. Airline Industry. Accounting Review, 68 (3), jul.1993. BROOKS, Cris. Introductory Econometrics for Finance. New York: Cambridge University Press, 2002.

CALLADO, Aldo Leonardo Cunha; CALLADO, Antônio André. Gestão de custos: apresentação de um modelo quantitativo sobre custos indiretos de produção. Anais... 2 ${ }^{\circ}$ Seminário de Controladoria e Contabilidade da USP. 2002.

DATAR, Srikant M. et al. Simultaneous Estimation of Cost Drivers. Accounting Review, 68 (3), jul.1993.

DICKEY, D. A.; FULLER, W. A.. Distribution of the estimator for auto-regressive time series with a unit root. Journal of the American Statistical Association, 74, 427-31, 1979.

Likelihood ratio statistics for autoregressive time series with a unit root. Econometrica, 49, 1057-1072, 1981.

FAVA, V. L.; CATI, R. C.. Mudanças no comportamento do PIB brasileiro: uma abordagem econométrica. Pesquisa e planejamento econômico, 25(2), ago, 1995.

GARRISON, Ray H.; NOREEN, Eric W. Contabilidade Gerencial. Rio de Janeiro: LTC, 2001.

GUJARATI, Damodar N. Econometria básica. São Paulo: Makron Books, 2000.

HILL, R. Carter.; GRIFFITHS, William E.; JUDGE, George G.. Econometria. Sao Paulo: Saraiva, 1999.

HORNGREN, Charles T.; FOSTER, George; DATAR, Srikant M. Contabilidade de Custos. Rio de Janeiro: LTC, 2000.

IUDÍCIBUS, Sérgio. Análise de Custos. São Paulo: Atlas, 1989.

KARMEL, Peter; POLASEK, M.. Estatística Geral e Aplicada a Economia. São Paulo: Atlas, 1981.

LEONE, George S. Guerra. Custos: Planejamento, implantação e Controle. São Paulo: Atlas, 2000.

MAHER, Michael. Contabilidade de Custos: Criando valor para a administração. São Paulo: Atlas, 2001.

MARTINS, Eliseu. Contabilidade de Custos. São Paulo: Atlas, 2003.

MEDEIROS, Otávio de. Testes Empíricos sobre o Comportamento Assimétrico dos Custos nas Empresas Brasileiras. Revista de Contabilidade \& Finanças - USP. 38. Maio/Ago. 2005.

SHANK, John K.; GOVINDARAJAN, Vijay. Gestão Estratégica de Custos: A nova ferramenta para a vantagem competitiva. Rio de Janeiro: Campus, 1995.

STEVENSON, William J. Estatística Aplicada a Administração. São Paulo: HABRA, 1981.

\section{NOTA - Endereço dos autores}

Universidade Federal de Pernambuco

Centro de Ciências Sociais Aplicadas

Departamento de Ciências Contábeis

Av. dos Professores, s/n. - Campus Universitário

Recife - PE

50670-901

Universidade de Brasília

Campus Universitário Darcy Ribeiro

Brasília-DF

70910-900
Fac. de Filosofia Ciênc. e Letras de Caruaru

R. Azevedo Coutinho, $\mathrm{s} / \mathrm{n}^{\circ}$. - Petrópolis

Caruaru - PE

55000-000 